\title{
Expression-Invariant 3D Face Recognition using Patched Geodesic Texture Transform
}

\author{
Farshid Hajati $^{1,2}$, Abolghasem A. Raie ${ }^{1}$ \\ ${ }^{1}$ Faculty of Electrical Eng., Amirkabir University of \\ Technology, Tehran, Iran \\ ${ }^{2}$ School of Engineering, Griffith University, QLD 4111, \\ Australia \\ hajati@aut.ac.ir, raie@aut.ac.ir
}

\author{
Yongsheng $\mathrm{Gao}^{2,3}$ \\ ${ }^{2}$ School of Engineering, Griffith University, QLD 4111, \\ Australia \\ ${ }^{3}$ Queensland Research Lab, National ICT Australia, Australia \\ yongsheng.gao@griffith.edu.au
}

\begin{abstract}
Numerous methods have been proposed for the expression-invariant 3D face recognition, but a little attention is given to the local-based representation for the texture of the 3D images. In this paper, we propose an expression-invariant 3D face recognition approach based on the locally extracted moments of the texture when only one exemplar per person is available. We use a geodesic texture transform accompanied by Pseudo Zernike Moments to extract local feature vectors from the texture of a face. An extensive experimental investigation is conducted using publicly available BU-3DFE face databases covering face recognition under expression variations. The performance of the proposed method is compared with the performance of two benchmark approaches. The encouraging experimental results demonstrate that the proposed method can be used for 3D face recognition in single model databases.
\end{abstract}

Keywords-3D face; expression; geodesic distance; texture

\section{INTRODUCTION}

Face recognition is a challenging task because of the variety in expression, age, pose, illumination, and occlusion [1]. 2D face recognition algorithms have a good performance under controlled conditions, but their performance reduces in the presence of condition variations. Recently, 3D information of the face has been used to tackle this problem. The proposed methods for 3D face recognition approaches can be divided into two categories: range approaches and range-texture approaches. The first category consists of methods which only use the features extracted from the range (depth) images for recognition. Cartoux et al. [2] used facial profiles extracted from range images for face recognition. Gordon [3] suggested the feature extraction based on depth and curvature features. Tanaka et al. [4] considered the face recognition problem as a $3 \mathrm{D}$ shape recognition problem of rigid surfaces. Their method is based on the surface curvature information. Chua et al. [5] extended the use of point signature to recognize frontal face scans with expression changes. Hesher et al. [6] applied PCA to the range images, and estimated the probability models for the coefficients.

The range-texture approaches are the algorithms which use both range (depth) and texture information to improve the recognition rate on $3 \mathrm{D}$ information. Change et al. [7] showed that the face recognition performances on texture and depth images are same. However, the combination of them can improve the recognition rate. Malassiotis and Strintzis [8] proposed a technique for face recognition using texture and depth information. They used the depth images to compensate pose variations in corresponding texture images. They compensated the pose of input face images by matching the input depth image with a reference model using the ICP algorithm [9]. The classification was performed using an Embedded Hidden Markov Model (EHMM) [10] on the compensated images. Lu et al. [11] used a matching technique between range face images and 3D face models for face recognition under pose variations. For each subject a reference 3D face model constructed by merging several face range images from different views. The recognition step consists of two components: a surface matching and an appearance matching. They used a modified ICP algorithm to match an input range image and the reference 3D models. Based on the surface matching's output, a candidates list from the gallery are used for the appearance matching. Bronstein et al. $[12,13]$ proposed an expression-invariant representation of faces called Canonical Images. The canonical images are built by calculating the geodesic distances between points on the face surface. Mpiperis et al. [14] proposed a geodesic polar representation of the face surface. They used a geodesicbased isometric mapping to provide some warped texture images and apply PCA technique for classification.

We present a novel 3D face recognition approach using Patched Geodesic Texture Transform (PGTT). In this approach, we use local texture moments controlled by the geodesic shape information to tackle the problems of face recognition under expression variations when only one exemplar per person is available. Instead of using the global texture representation, which is not effective under different facial variations, the proposed method employs a local texture representation. The feasibility and effectiveness of the proposed method is investigated using the standard publically available BU-3DFE face database [15] which contains faces under various situations including different expression changes. The performance of the system is compared with the performance of two benchmark 
approaches. Encouraging experimental results indicate that the proposed method provides a novel solution for expression-invariant face recognition using 3D images.

The rest of this paper is organized as follows: Section 2 presents the proposed Patched Geodesic Texture Transform. In Section 3, the proposed method is extensively examined and compared with two benchmark approaches. Finally, Section 4 concludes the paper.

\section{Proposed Patched Geodesic TeXture TRANSFORM}

In the proposed algorithm, we create the corresponding range and texture images from an input $3 \mathrm{D}$ image. Using the range image, we compute the geodesic distance from a reference point for all face points. Based on the computed distances, we transform the texture image to a new texture image called transformed texture image. In the next step, the transformed texture image is partitioned into some equalsized patches and a patch descriptor is applied to extract a feature vector for each patch. By concatenating the feature vectors, we build a feature vector for the subject. Finally, the input subject is identified by measuring the similarity between the query image and all gallery models. The following subsections will describe the algorithm in detail.

\section{A. Geodesic Distance Computing}

Let $S$ be a surface, $P$ and $Q$ two points on $S$. There are an infinite number of curves that belong to $S$ and connect $P$ with $Q$. The curve on the surface $S$ with the minimum length is called the Geodesic Path between two points and its length is called the Geodesic Distance. The computation of the geodesic distance on a surface involves the solving of Eikonal equation [16]

$$
|\nabla T(x, y, z)|=1
$$

on the surface, where $T(x, y, z)$ is the geodesic distance of the surface point $Q(x, y, z)$ from a reference point (e.g. $P$ ). The main approach to solve the Eikonal equation is the Fast Marching Method (FMM) [17]. In a 3D space we can compute the geodesic distance in the triangular meshes, consisting of vertexes and faces, using the Fast Marching Method on Triangulated Domains (FMTD) [18].

In this paper, we compute the geodesic distances on face surface using the range images extracted from the $3 \mathrm{D}$ face models. If we represent the range image as $z=f^{r}(x, y)$, in which the superscript $r$ stands for range, the Equation (1) can be solved on the range image as

$$
\left|\nabla T\left(x, y, f^{r}(x, y)\right)\right|=1
$$

where $T\left(x, y, f^{r}(x, y)\right)$ is the geodesic distance of the point $Q\left(x, y, f^{r}(x, y)\right)$ from the reference point. Here, we consider the nose tip as the reference point. The origin of the range image coordinates is located at bottom-left corner of the image.

\section{B. Texture Transform}

It has proved that the geodesic distance can be used to represent the texture and shape in the face recognition algorithms [13, 14, 19, 20]. Bronstein et al. [13] used the basic geodesic distance between all face points to create a Canonical Image Representation for the face texture. This approach relies on the assumption that the face is approximately isometric, which means that the geodesic distances among face points on the face surface are preserved in different expression changes. The Mpiperis et al. [14] proposed a Geodesic Polar Representation based on the geodesic paths and circles on the face surface. They used the geodesic circles and geodesic paths on the face surface to create geodesic polar coordinates.

In this paper, we use the notion of face isometric assumption to find a novel texture transform which is more robust to expression variations than the existing methods. Our transform is based on the assumption that the face surface is a 2D manifold embedded in a 3D Euclidean space. We can characterize the face surface by a Riemannian metric and describe it by geodesic properties. For this purpose, we need the texture image of the face. The texture image can be created by projecting the pixels of the $3 \mathrm{D}$ face model to the $\mathrm{x}-\mathrm{y}$ plane. The origin of the texture image coordinates is located at bottom-left corner of the image.

We represent the created texture image from the face 3D model as $I=f^{t}(x, y)$, in which the superscript $t$ stands for texture. Because the creating procedure for the texture image is exactly same as the creating procedure for the range image, for each pixel in the created texture image we have a geodesic distance computed from the range image. We use the geodesic distance of the face points to establish a Texture Transform for the texture image. The proposed texture transform of the point $(x, y)$ in the texture image is computed by the Equation (3).

where

$$
\mathcal{F}^{t}\left(x_{\text {trans }}, y_{\text {trans }}\right)=f^{t}(x, y)
$$

and

$$
x_{\text {trans }}=r\left(x, y, f^{r}(x, y)\right) \cos (\varphi(x, y))+80
$$

$$
y_{\text {trans }}=r\left(x, y, f^{r}(x, y)\right) \sin (\varphi(x, y))+70
$$

where $\mathcal{F}^{t}\left(x_{\text {trans }}, y_{\text {trans }}\right)$ denotes the transformed texture image in which the superscript $t$ stands for transformed. $x_{\text {trans }}$ and $y_{\text {trans }}$ are the transformed image coordinates of the point in the transformed texture image. The origin of the coordinates system in the transformed texture image is located at $(80,70) . r\left(x, y, f^{r}(x, y)\right)$ is the geodesic distance of the point $Q\left(x, y, f^{r}(x, y)\right)$ from the nose tip as

$$
r\left(x, y, f^{r}(x, y)\right)=T\left(x, y, f^{r}(x, y)\right)
$$

where $T$ is the geodesic distance computed by (2).

In Equations (4) and (5), $\varphi(x, y)$ is the angle of the point $Q(x, y)$ in the texture image respect to the reference point (nose tip). Mathematically, $\varphi(x, y)$ is defined as 


$$
\varphi(x, y)= \begin{cases}0 & \text { if } x=x_{R P} \\ \tan ^{-1}\left(\frac{y-y_{R P}}{x-x_{R P}}\right) & \text { if } x>x_{R P} \\ \tan ^{-1}\left(\frac{y-y_{R P}}{x-x_{R P}}\right)+\pi & \text { if } x<x_{R P}\end{cases}
$$

where $\left(x_{R P}, y_{R P}\right)$ is the location of the Reference Point (RP) in the texture image.

Finally, we crop the image to obtain a face rectangle which includes the most important facial features. For this purpose, a cropping model in that the distance of the nose tip is 80 from the sides, 90 and 70 from the top and the bottom, respectively, is used. Therefore, a $160 \times 160$ image is created in which the origin of the transformed image coordinates is located at bottom-left of the image. Fig. 1 depicts an example of a cropped transformed texture image using the above procedure. Fig. 1 illustrates an example of a face 3D model and its transformed texture image.
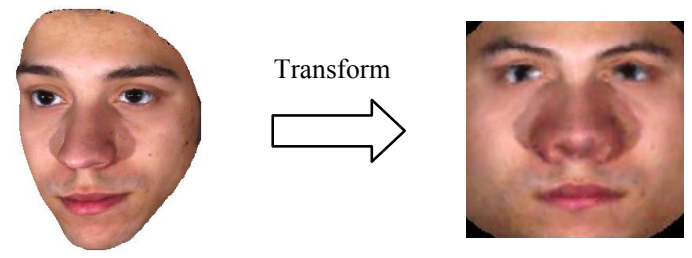

Figure 1. An example of a face 3D model and its transformation

\section{Descriptor}

In this paper, we use Pseudo Zernike Moments as descriptor. Pseudo Zernike polynomials are orthogonal sets of complex-valued polynomials [21]. It has proved that the PZM have the best performance for image representation among the common moments [22]. PZMs have been utilized as descriptor in a range of face recognition applications in expression and lighting changing conditions, partial occlusion, and from one exemplar per person.

PZM of order $n$ and repetition $m$ of a continuous intensity image $f(x, y)$ can be defined as

$$
\begin{aligned}
& \operatorname{PZM}_{n, m}(f(x, y)) \\
& =\frac{n+1}{\pi} \iint_{x^{2}+y^{2} \leq 1} V_{n, m}^{*}(x, y) f(x, y) d x d y
\end{aligned}
$$

where $n \geq 0,|m|<n$ and the symbol * denotes the complex conjugate. The Pseudo Zernike polynomials $V_{n, m}^{*}(x, y)$ are defined as

$$
V_{n, m}^{*}(x, y)=R_{n, m}(a) e^{j m \theta}
$$

where $a=\sqrt{x^{2}+y^{2}}$ is the length of the vector from the origin to the pixel $(x, y)$ and $\theta=\tan ^{-1}\left(\frac{y}{x}\right)$. The radial polynomials $R_{n, m}(a)$ are defined as

$$
R_{n, m}(a)=\sum_{s=0}^{n-|m|}(-1)^{s} \frac{(2 n+1-s) !}{s !(n-|m|-s) !(n+|m|+1-s) !} a^{n-s}
$$

In this paper, we use PZMs as a local descriptor in the proposed face recognition system. This descriptor is applied on the transformed texture images created in the previous

section to extract feature vectors. For this purpose, we first partitioned the transformed texture image to some equalsized patches in a non-overlapping way. Assume that the size of the image is $N \times N$ and the size of each patch is $W \times W$. In this case, the number of patches for the transformed texture image is $(N / W)^{2}$. We indicate all points in a patch with $p$ and $q$ indexes which are integers ranging from 1 to $N / W$ and mathematically can be found for each point as

$$
\begin{array}{ll}
p=\left\lfloor\frac{x_{\text {trans }}}{W}\right]+1 & ; \quad 0 \leq x_{\text {trans }}<N \\
q=\left\lfloor\frac{y_{\text {trans }}}{W}\right]+1 & ; \quad 0 \leq y_{\text {trans }}<N
\end{array}
$$

which $x_{\text {trans }}$ and $y_{\text {trans }}$ are transformed image coordinates. The symbol $[\cdot]$ denotes the floor function. Fig. 2 illustrates an example of partitioning a transformed texture image into patches.

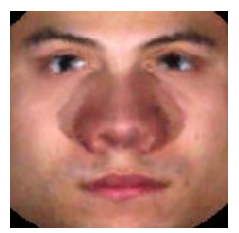

Figure 2. Partitioning a transformed texture image into patches

The $(p, q)$ th patch of the transformed texture image can be represented as

$$
\begin{aligned}
\mathcal{F}_{\text {pq }}^{t}\left(x_{\text {trans }}^{p q}, y_{\text {trans }}^{p q}\right) & \\
= & \mathcal{F}^{t}(W(p-1) \\
& \left.+x_{\text {trans }}^{p q}, W(q-1)+y_{\text {trans }}^{p q}\right)
\end{aligned}
$$

where $x_{\text {trans }}^{p q}$ and $y_{\text {trans }}^{p q}$ are the transformed patch coordinates of the $(p, q)$ th patch. The origin of the patch coordinates system is located at the bottom-left corner of each patch.

By substituting (13) in (8), we can find the PZM equation for the $(p, q)$ th patch in the transformed texture image as

$$
\begin{aligned}
& \operatorname{PZM}_{n, m}^{p q}\left(\mathcal{F}^{t}\left(x_{\text {trans }}, y_{\text {trans }}\right)\right) \\
& =\frac{n+1}{\pi} \iint_{x^{2}+y^{2} \leq 1} V_{n, m}^{*}(x, y) \mathcal{F}_{p, q}^{t}\left(x_{\text {trans }}^{\text {pq }}, y_{\text {trans }}^{\text {pq }}\right) d x d y
\end{aligned}
$$

where $p$ and $q$ are integers ranging from 1 to $N / W$.

To compute the PZM for each patch, the texture of the patch is mapped (normalized) to a unit circle. The approach that we use in this paper is the mapping of the patch in the way that the entire patch is bounded inside the unit circle. This approach ensures that there is no pixel loss during the PZM computations. We perform the normalization into the unite circle by the following equations:

$$
x_{\text {trans }, i}^{p q}=-\frac{\sqrt{2}}{2}+\frac{\sqrt{2}}{W-1} i \quad ; i=0,1, \ldots, W-1
$$




$$
y_{\text {trans, } j}^{p q}=\frac{\sqrt{2}}{2}-\frac{\sqrt{2}}{W-1} j \quad ; j=0,1, \ldots, W-1
$$

where $x_{\text {trans, } i}^{p q}$ and $y_{\text {trans }, j}^{p q}$ are normalized transformed patch coordinates of the points in the $(p, q)$ th patch. The origin of the normalized transformed patch coordinates system is located at the centre point of the patch.

The Equation (14) is the continuous form of the PZM. By using Equations (15) and (16) in (14) we can derive a discrete form of PZM compatible for the transformed image. The discrete form of PZM of order $n$ and repetition $m$ for the $(p, q)$ th patch in the transformed texture image is written as

$$
\begin{aligned}
& \operatorname{PZM}_{n, m}^{p q}\left(\mathcal{F}\left(x_{\text {trans }, i}, y_{\text {trans }, j}\right)\right) \\
& =\frac{n+1}{\pi \lambda(W)} \sum_{i=0}^{W-1} \sum_{i=0}^{W-1} \mathcal{F}_{n, m}^{*}\left(x_{\text {trans }, i}^{\text {pq }}, y_{\text {trans }, j}^{\text {pq }}\left(x_{\text {trans }, i}, y_{\text {trans }, j}^{\text {pq }}\right)\right.
\end{aligned}
$$

where $x_{\text {trans }, i}$ and $y_{\text {trans }, j}$ are normalized transformed coordinates in the transformed texture image. The normalization factor $\lambda(W)$ is the ratio between the number of pixels in the patch before and after normalizing into the unit circle. This normalization factor for our mapping is $W / 2$.

In this paper, the magnitudes of PZMs of order 1 up to $n_{\max }$ with repetition $m \geq 0$ will be considered as moment features. The $n_{\max }$ will be defined in section 3.1 by the experiment. The feature vector for the $(p, q)$ th patch can be represented as

$$
\begin{gathered}
P Z M_{n_{\max }^{p q}}^{p q}=\left\{\left|P Z M_{u, v}^{p q}\right| \mid u\right. \\
=1, \ldots, n_{\max } \text { and } v \\
=0, \ldots, u\}
\end{gathered}
$$

where the symbol $|\bullet|$ denotes the absolute value operator.

The output of the patch descriptor section is a feature vector for each patch in the transformed texture image. In the next section, we use these feature vectors to calculate the dissimilarity between two subjects.

\section{Dissimilarity Measurement}

In this section, we calculate the distance of a given query 3D image and a model in the gallery. We use the extracted PZMs to measure the dissimilarity between images. The Texture Distance Vector (TDV) between the $(p, q)$ th patch of the transformed texture images in the query and the model is defined as

$$
T D V_{p q}=\left\{\left[P Z M_{n_{\max }}^{p q}\right]_{f}-\left[P Z M_{n_{\max }}^{p q}\right]_{g}\right\}
$$

where $f$ and $g$ are the transformed texture images of the query and the model faces, respectively.

In order to decrease the effect of the boundary patches, patches located on the face border (see Fig. 2), on the extracted moments, an adaptively weighting technique is used to weight the moment features of each patch. For this purpose, we define the weight of each patch based on the patch area in the transformed query image and the corresponding patch area in the transformed model image.
We map both patches into the unit circle as the procedure described in the previous section and compute the proportion of the intersection area of two patches to the whole area as

$$
\gamma_{p q}=\frac{s_{p q}^{Q} \cap s_{p q}^{M}}{2}
$$

where $\gamma_{p q}$ is the weightage for the $(p, q)$ th patch, $S_{p q}^{Q}$ and $S_{p q}^{M}$ are the mapped area of the $(p, q)$ th patch of the transformed texture images in the query and transformed model images, respectively. The superscripts $Q$ and $M$ stand for query and model, respectively. The symbol $\cap$ denotes the intersection operator.

By using $T D V_{p q}$ and $\gamma_{p q}$, we can compute the distance between the query face $(f)$ and the model face $(g)$ as

$$
D(f, g)=\left\|\left\{\gamma_{p q} \bullet T D V_{p q} \mid p, q=1, \ldots, \frac{N}{W}\right\}\right\|
$$

where the symbol $\bullet$ denotes the mathematical multiplication.

For a given query image, we compute the distance to all models in the gallery using Equation (21). The model in the gallery with the minimum distance $(D)$ is considered as the correct return.

\section{EXPERIMENTAL RESULTS}

In order to evaluate the feasibility and effectiveness of the proposed approach, an extensive experimental investigation is conducted, covering face recognition under different facial expressions. The experiments were conducted on the BU-3DFE [15] which contains 3D face models under all different expression changes. The database contains 100 subjects (56 females, 44 males) and each subject has seven expressions. With the exception of the neutral expression, each of the six prototypic expressions (happiness, disgust, fear, angry, surprise and sadness) includes four levels of intensity. The performance of the proposed method is compared with two benchmark approaches: 1) the method proposed by Bronstein for expression-invariant face recognition (Canonical Image Representation) [13], 2) the method proposed by Mpiperis for expression-invariant face recognition (Geodesic Polar Representation) [14].

\section{A. Determination of Parameters}

In this section, we determine two parameters involved in the proposed method, i.e. the maximum order of the PZMs $\left(n_{\max }\right)$ and the size of the patches $(W)$. In theory, the PZM with order zero represents the DC component (mean value of the pixels in the image) and do not carry personal identity information. Hence, we set the minimum order of the PZMs as 1 .

To determine $n_{\max }$ and $W$, an experimental investigation on recognition accuracy is conducted on a training dataset. For this purpose, we created a training dataset from the BU-3DFE database [15]. For each subject, we selected two faces: one is the neutral face and another is one of the six prototypic expressions with one of four 
expression levels selected randomly. In the training set, all neutral faces are selected as the gallery and others are used as the probe. In this experiment, we change the size of the patch $(W)$ in the range of $80,40,32,20,16$, and 10. For each patch size, we measure the accuracy of the system for different values of the maximum order of the PZMs $\left(n_{\max }\right)$ and draw the corresponding curve. The result is displayed in Fig. 3.

It can be observed that through reducing the patch size, system accuracy continuously increased and reached the highest rate when $\mathrm{W}=16$, then decreased when $\mathrm{W}=10$. It is encouraging to see that the required $n_{\max }$ (i.e. the value of $n_{\max }$ when the curve starts to remain flat) continuously decreases when $\mathrm{W}$ decreases. In the rest of experiments, we choose $\mathrm{W}=16$ as the patch size, and $n_{\max }=3$ as the maximum order of PZM.

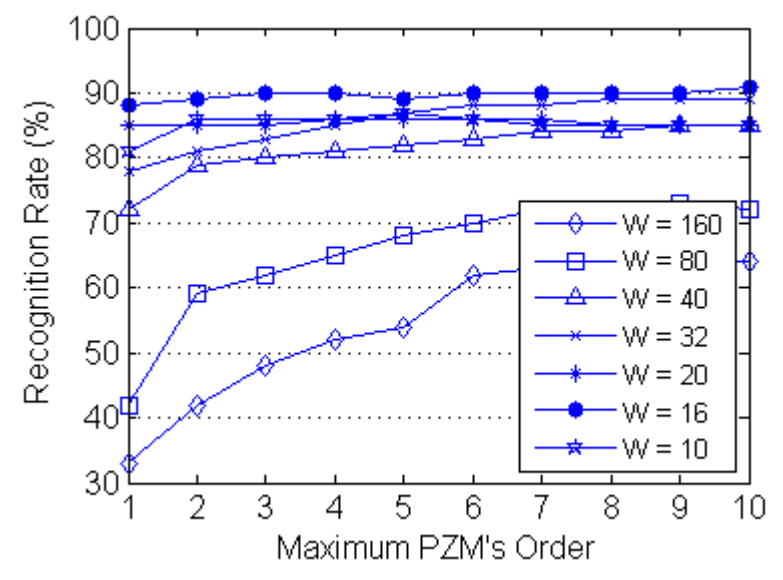

Figure 3. The effect of $W$ and $n_{\max }$ on the recognition rate

\section{B. Face Recognition}

The robustness to facial expression variation is an important issue in a face recognition system. Some experiments were conducted to evaluate the effects of different facial expressions (angry, disgust, fear, happy, sad, and surprise) on the proposed approach. To have more reliable comparison with the results of benchmarks reported in [14], the same experiment strategy used in [14] is adopted in our study. The performance is measured in term of the rank-1 recognition rate [23]. For each person, a single neutral model is used as representative in the gallery, while the rest images depicting expressions are used as probe. In total we have seven probe sets: six probe sets for the recognition rate under six different face expression changes (angry, disgust, fear, happy, sad, and surprise) and one probe set for the recognition on whole database.

The rank-1 recognition rates of the proposed algorithm for each type of the expression changes and for whole database are tabulated in table I. For comparison, the rank-1 recognition rates for the benchmarks on whole database are reported from [14]. As can be seen, the angry expression has the best accuracy $(90 \%)$, while the disgust expression has the worst accuracy (78\%). The overall accuracy for the proposed method $(82.2 \%)$ is higher than the benchmark approaches $(80.3 \%$ and $77.2 \%$ for Geodesic Polar Representation and Canonical Image Representation, respectively).

TABLE I. PERFROMANCE COMPARISON UNDER EXPRESSION CHANGES

\begin{tabular}{|cccc|}
$\begin{array}{c}\text { Expression } \\
\text { Considered }\end{array}$ & $\begin{array}{c}\text { The } \\
\text { Proposed } \\
\text { Method }\end{array}$ & $\begin{array}{c}\text { Geodesic Polar } \\
\text { Representation }\end{array}$ & $\begin{array}{c}\text { Canonical } \\
\text { Image } \\
\text { Representatio } \\
\text { n }\end{array}$ \\
Angry & 90 & N/A & N/A \\
Disgust & 78 & N/A & N/A \\
Fear & 80 & N/A & N/A \\
Happy & 82 & N/A & N/A \\
Sad & 82 & N/A & N/A \\
Surprise & 81 & N/A & N/A \\
Overall & 82.2 & 80.3 & 77.2 \\
\hline
\end{tabular}

\section{CONCLUSION}

In this paper, a novel expression-invariant face recognition method is proposed called Patched Geodesic Texture Transform (PGTT). PGTT is particularly designed to handle the variations in face surface due to the expression. The Pseudo Zernike Moments are adapted to use in the proposed face recognition algorithm as moments. The algorithm has been evaluated and compared with two benchmarks. It is very encouraging finding that the PGTT performs consistently superior to the benchmarks under the expression variations. This research reveals that the PGTT provides a new solution for expression-invariant face recognition.

\section{REFERENCES}

[1] A. F. Abate, M. Nappi, D. Riccio, and G. Sabatino, "2d and 3d face recognition: A survey," Pattern Recognition Letters, vol. 28, no. 14, pp. 1885-1906, 2007.

[2] J. Y. Cartoux, J. T. LaPreste, and M. Richetin, "Face authentication or recognition by profile extraction from range images," Proc. of the Workshop Interpretation of 3D Scenes, pp. 194-199, 1989.

[3] G. G. Gordon, "Face recognition based on depth and curvature features," Proc. of the IEEE Conference on Computer Vision and Pattern Recognition, pp. 108-110, 1992.

[4] H. T. Tanaka, M. Ikeda, and H. Chiaki, "Curvature-based face surface recognition using spherical correlation-principal directions for curved object recognition," Proc. of the 3rd. International Conference on Face and Gesture Recognition, pp. 372-377, 1998.

[5] C. Chua, F. Han, and Y. Ho, "3d human face recognition using point signature," Proc. of the 4th International Conference on Automatic Face and Gesture Recognition, pp. 233-238, 2000.

[6] C. Hesher, A. Srivastava, and G. Erlebacher, "Principal component analysis of range images for facial recognition," Proc. of the International Multiconference on Computer Science and Information Systems, pp. 62-68, 2002. 
[7] K. Chang, K. Bowyer, and P. Flynn, "Multi-modal 2d and 3d biometrics for face recognition," Proc. of the IEEE International Workshop on Analysis and Modeling of Faces and Gestures, pp. 187-195, 2003.

[8] S. Malassiotis, and M. G. Strintzis, "Robust face recognition using 2d and 3d data: Pose and illumination compensation," Pattern Recognition, vol. 38, no. 12, pp. 2537-2548, 2005.

[9] P. Besl, and N. McKay, "A method for registration of 3d shapes," IEEE Trans. on Pattern Analysis and Machine Intelligence, vol. 14, no. 2, pp. 239-256, 1992.

[10] L. R. Rabiner, "Tutorial on hidden markov models and selected applications in speech recognition," Proc. IEEE, vol. 77, no. 2, pp. 267-296, 1989.

[11] X. Lu, A. K. Jain, and D. Colbry, "Matching 2.5d face scans to $3 \mathrm{~d}$ models," IEEE Trans. on Pattern Analysis and Machine Intelligence, vol. 28, no. 1, pp. 31-43, 2006.

[12] A. M. Bronstein, M. M. Bronstein, and R. Kimmel, "Expression-invariant 3d face recognition," Proc. Audio-and Video-Based Biometric Person Authentication, vol. 2688, no. 1, pp. 62-69, 2003.

[13] A. M. Bronstein, M. M. Bronstein, and R. Kimmel, "Expression-invariant representations of faces," IEEE Trans. on Image Processing, vol. 16, no. 1, pp. 188-197, 2007.

[14] I. Mpiperis, S. Malassiotis, and M. G. Strintzis, "3-d face recognition with the geodesic polar representation," IEEE Trans. on Information Forensics and Security, vol. 2, no. 3, pp. 537-547, 2007.

[15] L. Yin, X. Wei, Y. Sun, J. Wang, and M. J. Rosato, "A 3d facial expression database for facial behavior research," Proc. of the 7th International Conference on Automatic Face and Gesture Recognition (FGR), pp. 211-216, 2006.

[16] Y. R. Tsai, L. Cheng, S. Osher, and H. Zhao, "Fast sweeping algorithms for a class of hamilton-jacobi equations," SIAM Journal on Numerical Analysis, vol. 41, no. 2, pp. 673-694, 2003.

[17] J. A. Sethian, Level set methods and fast marching methods evolving interfaces in computational geometry, Fluid mechanics, computer vision, and materials science, Cambridge: Cambridge University Press, 1999.

[18] R. Kimmel, and J. A. Sethian, "Computing geodesic paths on manifolds," Applied Mathematics, vol. 95, no. 15, pp. 84318435, 1998.

[19] A. M. Bronstein, M. M. Bronstein, R. Kimmel, and A. Spira, "Face recognition from facial surface metric," Proc. of the 8th European Conference on Computer Vision, pp. 225-237, 2004.

[20] A. M. Bronstein, M. M. Bronstein, and R. Kimmel, "Threedimensional face recognition," International Journal of Computer Vision, vol. 64, no. 1, pp. 5-30, 2005.

[21] M. R. Teague, "Image analysis via the general theory of moments," Journal of the Optical Society of America, vol. 70, no. 1 , pp. 920-930, 1980.

[22] P. Tzouveli, K. Ntalianis, and S. Kollias, "Human face watermarking based on zernike moments," Proc. of the Fifth IEEE International Symposium on Signal Processing and Information Technology, pp. 399-404, 2005.

[23] S. A. Rizvi, P. J. Phillips, and H. Moon, "The feret verification testing protocol for face recognition algorithms," Proc. of the International Conference on Face and Gesture Recognition, pp. 48-53, 1998. 